\title{
Perianal fistula imaging: a comparison between two-channel superficial Flex coil and eight-channel body coil
}

\author{
Nazlı Gülsüm Akyel ${ }^{1 \mathrm{AB}, \mathrm{B}, \mathrm{E}, \mathrm{F}}$, Kayıhan Akın ${ }^{2 A, D, F}$, Dilek Kösehan ${ }^{3 B, D}$, Aslı Köktener ${ }^{3 B, F}$ \\ 'Cerrahpaşa Medical Faculty, Istanbul University, Istanbul, Turkey \\ ¿Lokman Hekim Akay Hospital, Ankara, Turkey \\ ${ }^{3}$ Memorial Ankara Hospital, Ankara, Turkey
}

\section{Abstract}

Purpose: Perianal fistula is an important health problem with devastating morbidity. For diagnosis and prevention of recurrence, perianal fistula imaging (PFI) is crucial. The aim of this study was to investigate if a Flex-M superficial coil images would provide more information than images obtained with a phased-array body coil in PFI.

Material and methods: Thirty-one fistulas of 29 patients were prospectively evaluated with magnetic resonance imaging. A phased-array body coil was used in all patients as in routine practice, and sequences with Flex-M coil were also obtained. Afterwards, images were evaluated by two experienced radiologists who were blinded to the patients, coil and also to each other. The site of internal and external openings, and presence or absence of abscess and secondary tracts were recorded.

Results: The conspicuity of images was better with the Flex-M coil. Both radiologists saw more internal openings with the Flex-M coil. Cohen's $\kappa$ value was $0.100(p=0.201)$ for Reader 1 and $0.110(p=0.361)$ for Reader 2 between these two coils. Additionally, numerically more internal/external openings and secondary tracts were seen by both readers. Interobserver variability was not statistically significant, and Cohen's $\kappa$ values signifyied good concordance between readers.

Conclusions: In our study we showed that multichannel superficial phased-array coils improved imaging quality in PFI. We think that Flex-M coil can easily be used in routine clinical practice to gather better images of perianal fistulas.

Key words: magnetic resonance imaging, coil, perianal fistulae.

\section{Introduction}

Perianal fistula is an important health problem with devastating morbidity, including severe local pain, chronic perianal discharge, impairment of social activities, and incontinence [1]. It is defined as an epithelial-lined abnormal tract between the anal canal and the skin [2]. Its exact prevalence is not known, but it is estimated to be around $1: 10000$. The male-to-female ratio is $2: 1$ [3].

Treatment is surgical, but due to missed infection sites, it has a tendency to recur; in some series it has had a recurrence rate of up to $25 \%$ [4]. Accurate preoperative assess- ment of primary perianal fistula and its relationship with sphincters, secondary tracks, and abscess formation is crucial for the establishment of a treatment plan. Besides its diagnostic purposes, magnetic resonance imaging (MRI) has become the gold standard method for perianal fistula imaging because it gives the most detailed information about the anatomical features of the fistula tract and its relationship with the anal sphincter muscles. By providing these data, MRI increases the rate of surgical success and decreases the recurrence rate of perianal fistula [2].

There are many publications about different magnetic resonance sequences with or without contrast material

Correspondence address:

Nazlı Gülsüm Akyel, MD, Department of Pediatric Radiology, Istanbul University - Cerrahpasa Medical Faculty, Cerrahpasa Mah. Kocamustafapasa Cad. No: 53,

34908 Fatih, Istanbul, Turkey, phone: +905345757708, e-mail: nazligulsumakyel@gmail.com

Authors' contribution:

A Study design · B Data collection · C Statistical analysis · D Data interpretation - E Manuscript preparation · F Literature search · G Funds collection 
[5-9] and diffusion-weighted MRI [10-13]. Additionally, endoanal coils and body coils have previously been compared with each other [14] or by surgical correlation. In the literature, it is noteworthy that some of the studies used torso [15-17] or spine coils [18]. However, there has been no published literature comparing body coils and superficial coils so far. Therefore, in our present study, we aimed to investigate whether using a two-channel Flex-M superficial coil would give more information compared to the images obtained with an eight-channel phased-array body coil in perianal fistula imaging.

\section{Material and methods}

\section{Patients}

All patients with anal fistula were planned to be involved in our present study. The presence of malignancy was accepted as an exclusion criterion.

All fistulas were idiopathic except in one patient with Crohn's disease. All patients were informed about the study, and the patients who agreed to be involved in the study were recruited accordingly. Our study was approved by the Local Ethics Committee, and informed consent was obtained from each study participant.

\section{Imaging technique}

Patients underwent 1.5 T MRI imaging (Achieva; Philips Medical Systems; Best, Netherlands) in the supine position. Each examination included sagittal fat-suppressed (FS) T2-weighted (T2W) (time of repetition/time of echo [TR/TE], 3500/80 ms), coronal FS T2W (TR/TE, $3500 / 80 \mathrm{~ms}$ ), axial T1W (TR/TE, 550/7 ms), T2W (TR/ TE, $4500 / 80 \mathrm{~ms}$ ), FS T2W (TR/TE, 4500/80 ms), FS T1W (TR/TE, 550/7 ms), axial dynamic THRIVE (TR/ TE, 4.6/2.2 ms), postcontrast axial, sagittal, and coronal FS T1W (TR/TE, 475-650/7 ms) images. Other imaging parameters included a slice thickness of $3.5 \mathrm{~mm}$ with an interslice gap of $1 \mathrm{~mm}$, a matrix size of $175 \times 385$, and a field of view (FOV) of $240 \times 240 \mathrm{~mm}$.

Distal rectum and skin were included. Axial and coronal imaging was performed as oblique axial and oblique coronal parallel to the anal canal (true axial and true coronal). Postcontrast series were obtained following bolus intravenous injection of $0.1 \mathrm{mmol} / \mathrm{kg}$ MRI contrast agent (Gadoversetamid, Optimark, Covidien).

All MRI investigations were performed with a phasedarray body coil. Additionally, an axial FS T2W sequence was gathered with Sense Flex-M coil (Achieva; Philips Medical Systems; Best, Netherlands) as a superficial coil. Patients were evaluated at a workstation. Afterwards, the axial FS T2W sequences that were previously obtained with Flex-M and body coils were evaluated separately by two experienced radiologists, who were blinded to the patients. Interpretations of the images by the two radiolo- gists were made at different times. The radiologists were unaware of each other's interpretations.

Flex and body coils were connected to the patient at the same time. The Flex coils were placed at the front and back of the patient's anal region, and a body coil was wrapped around it.

The site of internal and external openings and the presence of any abscess and secondary tracts were evaluated. Cases were categorised according to Park's [19] and St. James University [20] classification systems.

\section{Statistical analysis}

All statistical analyses were performed using the SPSS 21.0 Statistical Package Program for Windows (SPSS Inc., Chicago, IL, USA). Categorical variables were compared with the $\chi^{2}$ test. Intra- and inter-observer concordance were tested by Cohen's $\kappa$ test. $P$ values $<0.05$ were accepted as statistically significant.

\section{Results}

A total of 29 patients with 31 fistulas were included in our present study. Of the patients, 19 were male and 10 were female. The mean age of the study group was 42.8 years (age range: 19-68).

Reader 1 . Twenty-seven external openings of the fistulas could be seen by MRI with body coil, whereas 29 external openings could be seen by MRI with Flex-M coil. Cohen's $\kappa$ value for MRI with body coil and Flex-M coil was $0.635(p<0.001)$.

By MRI with body coil, 19 internal openings of the fistulas could be seen, whereas 30 internal openings could be seen by MRI with Flex-M coil. Cohen's $\kappa$ value was 0.100 $(p=0.201)$ for the two techniques (Table 1$)$. Six secondary fistula tracts were reported with body coil, whereas eight secondary fistula tracts were reported with Flex-M coil.

Reader 2. Twenty-five external openings could be seen by MRI with body coil, whereas 27 external openings could be seen by MRI with Flex-M coil. Cohen's $\kappa$ value for MRI with body coil and Flex-M coil was 0.763 $(p<0.001)$.

Eighteen internal openings could be seen by MRI with body coil, whereas 28 internal openings could be seen by MRI with Flex-M coil. Cohen's $\kappa$ value for MRI with body coil and Flex-M coil was $0.110(p=0.361)$.

Six secondary fistula tracts were reported with body coil, whereas eight secondary fistula tracts were reported with Flex-M coil. Cohen's $\kappa$ value for MRI with body coil and Flex-M coil was $0.817(p<0.001)$.

There were six abscesses in six different patients, and all these abscesses were reported by both readers with both imaging techniques.

A second fistula was seen in two different patients. Neither reader could see the second fistulas by MRI with body coil. However, Reader 1 reported the second fistula 
Table 1. Power of magnetic resonance imaging with Flex-M and body coil for visualisation of external opening, internal opening, secondary tract, and abscess related with perianal fistula

\begin{tabular}{|c|c|c|c|c|}
\hline & \multicolumn{2}{|c|}{ Reader 1} & \multicolumn{2}{|c|}{ Reader 2} \\
\hline & $\begin{array}{l}\text { Flex-M } \\
\text { coil }\end{array}$ & $\begin{array}{c}\text { Body } \\
\text { coil }\end{array}$ & $\begin{array}{l}\text { Flex-M } \\
\text { coil }\end{array}$ & $\begin{array}{c}\text { Body } \\
\text { coil }\end{array}$ \\
\hline \multicolumn{5}{|l|}{ External opening } \\
\hline Patients, $n(\%)$ & $29(93.5)$ & $27(87.1)$ & 27 (87.1) & $25(80.6)$ \\
\hline Cohen's $\kappa$ value* & \multicolumn{2}{|c|}{$0.635(p<0.001)$} & \multicolumn{2}{|c|}{$0.763(p<0.001)$} \\
\hline \multicolumn{5}{|l|}{ Internal opening } \\
\hline Patients, $n(\%)$ & $30(96.8)$ & $19(61.3)$ & $28(90.3)$ & $18(58.1)$ \\
\hline Cohen's $\kappa$ value* & \multicolumn{2}{|c|}{$0.100(p=0.201)$} & \multicolumn{2}{|c|}{$0.110(p=0.361)$} \\
\hline \multicolumn{5}{|l|}{ Secondary tract } \\
\hline Patients, $n(\%)$ & $8(25.8)$ & $6(19.4)$ & $8(25.8)$ & $6(19.4)$ \\
\hline Cohen's $\kappa$ value* & \multicolumn{2}{|c|}{$0.817(p<0.001)$} & \multicolumn{2}{|c|}{$0.817(p<0.001)$} \\
\hline \multicolumn{5}{|l|}{ Abscess } \\
\hline Patients, $n(\%)$ & $6(19.4)$ & $6(19.4)$ & $6(19.4)$ & $6(19.4)$ \\
\hline Cohen's $\kappa$ value* & \multicolumn{2}{|c|}{$1.000(p<0.01)$} & \multicolumn{2}{|c|}{$1.000(p<0.01)$} \\
\hline
\end{tabular}

*Kappa values show concordance between MRI with Flex-M coil and MRI with body coil

of both patients and Reader 2 reported one of them by MRI with Flex-M coil.

Inter-observer variability for the interpretation of the MRI images with Flex-M coil and body coil was not statistically significant, and kappa values signified good concordance between the readers (Table 2).

According to Park's classification, it was seen that $16(51.6 \%)$ patients had intersphincteric fistulas, 14 (45.2\%) had transsphincteric fistulas, and one (3.2\%) had suprasphincteric fistulas. No extrasphincteric fistulas were detected.

According to St. James University Hospital classification, 13 (41.9\%) patients had grade I fistulas, three (9.7\%) had grade II fistulas, eight (25.8\%) had grade III fistulas, six $(19.4 \%)$ had grade IV fistulas, and one (3.2\%) had a grade $\mathrm{V}$ fistula.

\section{Discussion}

In this study, we found that MRI could clearly depict perianal fistulas, abscess, secondary tracts, and their connections with sphincters. According to our results, the conspicuity of fistulas was better with fat-suppressed T2-weighted images obtained by MRI with superficial Flex-M coil, compared to those with body coil. This difference was more remarkable in showing the internal opening of the fistula tract (Figures 1 and 2). Besides this, there was excellent inter-observer concordance, which underlined that the operator dependency was low (Table 2).

Inadequate and/or false evaluation of the internal and external openings, primary and secondary tracts, unexpected anatomy, and concomitant inflammatory bowel disease are among the possible causes of surgical treat-
Table 2. Interobserver variability according to different imaging findings

\begin{tabular}{|l|c|c|}
\hline Variable & K value* $^{*}$ & $P$ value \\
\hline Internal opening imaging with Flex-M coil & 0.475 & 0.002 \\
\hline Internal opening imaging with body coil & 0.933 & $<0.001$ \\
\hline External opening imaging with Flex-M coil & 0.635 & $<0.001$ \\
\hline External opening imaging with body coil & 0.763 & $<0.001$ \\
\hline Secondary tract visualization with Flex-M coil & 1.000 & $<0.001$ \\
\hline Secondary tract visualization with body coil & 1.000 & $<0.001$ \\
\hline
\end{tabular}

*Kappa value shows the concordance between Reader 1 and Reader 2

ment failure [21]. It has been widely expressed that recurrence is inevitable if the correct internal opening is not identified and dealt with.

There are various imaging modalities for the evaluation of perianal fistulas. All these imaging techniques have been compared in various studies. For example, Buchanan et al. compared the accuracy of digital examination, endosonography, and MRI in the preoperative assessment of perianal fistula. In their study, they concluded that endosonography was superior to digital examination, but MRI remained superior in all aspects [22]. Besides this, MRI was shown to be very helpful for physicians in the establishment of a surgery plan before anal fistula operation [18] and in reducing relapses by up to $75 \%$ in complex cases [23]. Other techniques such as fistulography and computed tomography $(\mathrm{CT})$ proved no better than clinical examination [20-24]. Fistulography has been shown to be inaccurate sometimes [25], and CT has been shown to be hindered by tissue contrast [26].

Anal endosonography is superior to clinical examination [27], but it has an inadequate field of view (FOV) and poor patient tolerance. Alabiso et al. compared the performances of 3D-endoanal ultrasonography and MRI in the perianal fistulas of patients with Crohn's disease [28]. In their study, they proposed that MRI was preferable for the detection of supra- and extra-sphincteric fistulas and 3D-USG was preferable in the evaluation of inter-sphincteric fistulas. Significant operator dependence and patient discomfort are known to be important disadvantages of endoanal USG. CT fistulography is another imaging modality, but it is not routinely suggested in anal fistula imaging because it has some limitations in the differentiation of fistula tract, fibrosis, and sphincter muscles [24].

Although each imaging modality has its advantages and disadvantages, MRI is suggested by many experts as the gold standard method for perianal fistula imaging $[29,30]$. MRI is superior to other imaging techniques due to its excellent soft tissue contrast, operator independence, multiplanar capabilities, and superior FOV [31]. Accuracy rate of MRI in perianal fistula disease differs between studies and ranges between 86 and 100\% [24,32-35].

The optimal MRI technique for perianal fistula imaging is still a matter of debate. In a recent study, it was shown 


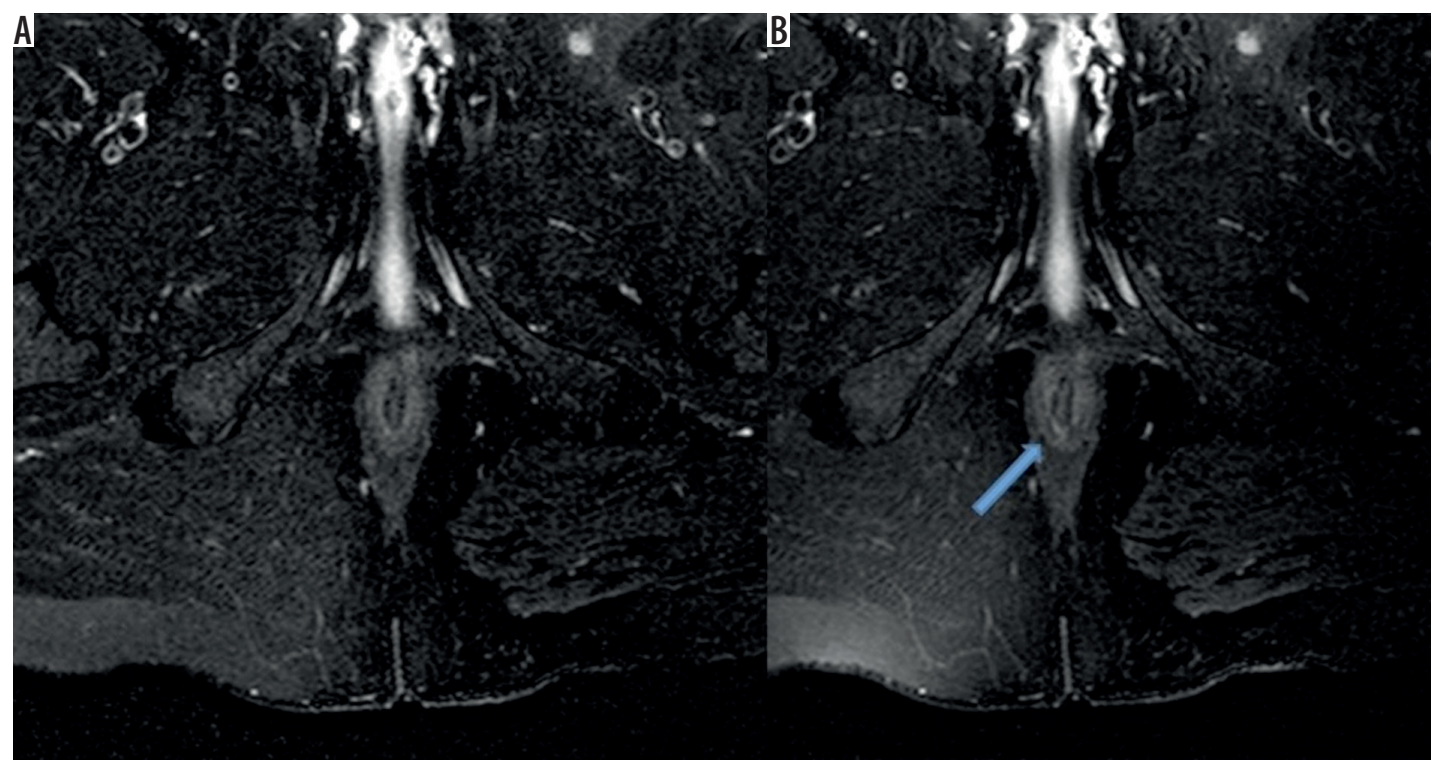

Figure 1. Twenty-nine-year-old female patient with recurrent fistula. Grade III transsphincteric fistula was seen on axial magnetic resonance images. Axial fat-saturated T2-weighted images obtained with body (A) and Flex-M coils (B). Internal opening was clearer in the image on the right side (arrow)

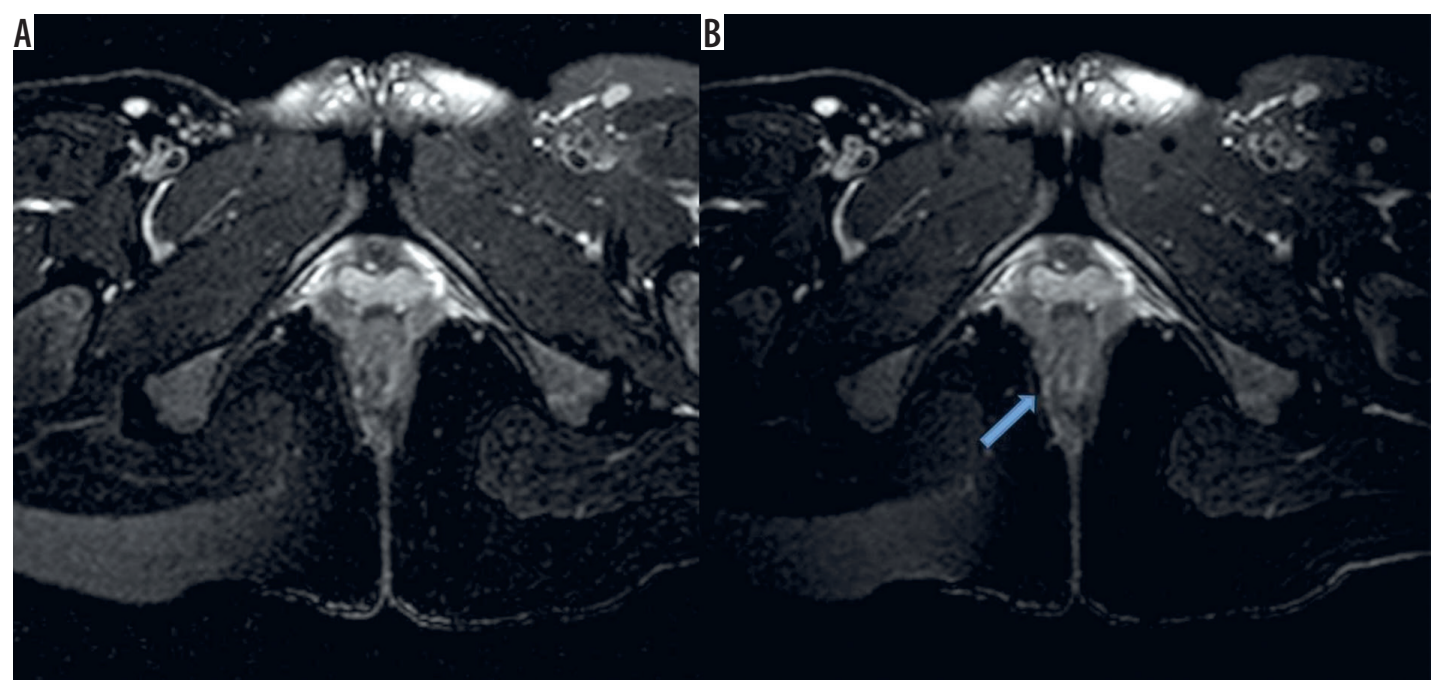

Figure 2. Forty-one-year-old male patient with grade I intersphincteric fistula. Axial fat-saturated T2-weighted images obtained with body (A) and Flex-M coils (B). Internal opening was clearly depicted on right picture (arrow)

that short-tau inversion recovery (STIR) sequences could be a valid alternative to post-contrast T1-weighted sequences in the MRI of perianal fistulas in Crohn's disease [1]. In another study, Baik et al. compared T2-weighted imaging (T2W), diffusion-weighted imaging (DWI), and contrast-enhanced T1-weighted imaging (CE-T1W), and according to their findings, they concluded that although DWI and CE-T1W improved fistula conspicuity they had comparable diagnostic performance with T2W [5]. Hori et al. investigated the additional value of DW MRI to T2W in the evaluation of perianal fistula disease in comparison with CE-T1W. In their study, they defined DWI as a useful sequence and proposed that it could be a helpful addition to T2W [10]. Due to high spatial resolution, endoanal coils were used in MR imaging as an alternative to body coils for better perianal fistula visualisation. However, surgical concordance remained at $68 \%$ for endoanal imaging, whereas it was $96 \%$ for MRI with conventional body coil imaging [36]. Patient discomfort and FOV limitation were among the most significant limitations of endoanal imaging.

Pelvic phased-array coils are preferred, because it has been demonstrated in recent studies that they provide higher resolutions. MRI acquisition quality has been improved by multichannel external phased-array coils. With these coils the acquisition time is decreased, signal-tonoise ratio is increased, and larger fields of view are provided without the need for endoanal coils [32]. Because of these advantages, we thought to examine whether using a superficial coil in routine MRI would give better results than conventional MRI with body coil. 
Our study does have some limitations. The number of patients was relatively low, and only axial FS T2W images were repeated with Flex-M coil because it would prolong the examination time. Thus, comparison of the coils could only be made based on the T2W images.

As a result, we showed that MRI with Flex-M coil gave clearer images and yielded additional data compared to perianal fistula imaging by MRI with body coil. Relatively easy accessibility and low cost are thought to be the most important advantages of Flex-M coil, making it preferable in perianal fistula imaging.
In conclusion, because proper visualisation of perianal fistulas is very important in the establishment of the treatment plan, we believe that our findings are crucial and applicable to routine daily clinical practice. MRI with Flex-M coil improves imaging quality in perianal fistula imaging and can easily be used in routine daily clinical practice.

\section{Conflict of interest}

The authors report no conflict of interest.

\section{References}

1. Lo Re G, Tudisca C, Vernuccio F, et al. MR imaging of perianal fistulas in Crohn's disease: sensitivity and specificity of STIR sequences. Radiol Med 2016; 121: 243-251.

2. Seow-Choen, Phillips RK. Insights gained from the management of problematical anal fistulae at St. Mark's Hospital, 1984-88. Br J Surg 1991; 78: 539-541.

3. Sainio P. Fistula-in-ano in a defined population: incidence and epidemiological aspects. Ann Chir Gynaecol 1984; 73: 219-224.

4. Lilius HG. Fistula-in-ano, an investigation of human foetal anal ducts and intramuscular glands and a clinical study of 150 patients. Acta Chir Scand Suppl 1968; 383: 7-88.

5. Baik J, Kim SH, Lee Y, et al. Comparison of T2-weighted imaging, diffusion-weighted imaging and contrast-enhanced T1-weighted MR imaging for evaluating perianal fistulas. Clin Imaging 2017; 44 16-21.

6. Beckingham IJ, Spencer JA, Ward J, et al. Prospective evaluation of dynamic contrast enhanced magnetic resonance imaging in the evaluation of fistula in ano. Br J Surg 1996; 83: 1396-1398.

7. Spencer JA, Ward J, Beckingham IJ, et al. Dynamic contrast-enhanced MR imaging of perianal fistulas. AJR Am J Roentgenol 1996; 167: 735-741.

8. Yıldırım N, Gökalp G, Ozturk E, et al. Ideal combination of MRI sequences for perianal fistula classification and the evaluation of additional findings for readers with varying levels of experience. Diagn Interv Radiol 2012; 18: 11-19.

9. Ziech ML, Lavini C, Bipat S, et al. Dynamic contrast-enhanced MRI in determining disease activity in perianal fistulizing Crohn disease: a pilot study. AJR Am J Roentgenol 2013; 200: W170-W177.

10. Hori M, Oto A, Orrin S, et al. Diffusion-weighted MRI: a new tool for the diagnosis of fistula in ano. J Magn Reson Imaging 2009; 30: 1021-1026.

11. Bakan S, Olgun DC, Kandemirli SG, et al. Perianal fistula with and without abscess: assessment of fistula activity using diffusionweighted magnetic resonance imaging. Iran J Radiol 2015; 12: e29084.

12. Cavusoglu M, Duran S, Sözmen CD, et al. Added value of diffusion-weighted magnetic resonance imaging for the diagnosis of perianal fistula. Diagn Interv Imaging 2017; 98: 401-408.

13. Yoshizako T, Wada A, Takahara T, et al. Diffusion-weighted MRI for evaluating perianal fistula activity: feasibility study. Eur J Radiol 2012; 81: 2049-2053.
14. deSouza NM, Gilderdale DJ, Coutts GA, et al. MRI of fistula-in-ano: a comparison of endoanal coil with external phased array coil techniques. J Comput Assist Tomogr 1998; 22: 357-363.

15. Waniczek D, Adamczyk T, Arendt J, et al. Direct MRI fistulography with hydrogen peroxide in patients with recurrent perianal fistulas: a new proposal of extended diagnostics. Med Sci Monit 2015; 21 : 439-445.

16. Ozkavukcu E, Haliloglu N, Erden A. Frequencies of perianal fistula types using two classification systems. Jpn J Radiol 2011; 29: 293-300.

17. Haliloglu N, Gulpinar B, Ozkavukcu E, et al. Typical MR imaging findings of perianal infections in patients with hematologic malignancies. Eur J Radiol 2017; 93: 284-288.

18. Beets-Tan RG, Beets GL, van der Hoop AG, et al. Preoperative MR imaging of anal fistulas: does it really help the surgeon? Radiology 2001; 218: 75-84.

19. Parks AG, Gordon PH, Hardcastle JD. A classification of fistula-in-ano. Br J Surg 1976; 63: 1-12.

20. Morris J, Spencer JA, Ambrose NS. MR imaging classification of perianal fistulas and its implications for patient management. Radiographics 2000; 20: 623-635.

21. Abou-Zeid AA. Anal fistula: Intraoperative difficulties and unexpected findings. World J Gastroenterol 2011; 17: 3272-3276.

22. Buchanan G, Halligan S, Bartram CI, et al. Clinical examination, endosonography, and MR imaging in preoperative assessment of fistula in ano: comparison with outcome-based reference standard Radiology 2004; 233: 674-681.

23. Buchanan G, Halligan S, Williams A, et al. Effect of MRI on clinical outcome of recurrent fistula-in-ano. Lancet 2002; 360: 1661-1662.

24. Halligan S, Jaap S. Imaging of fistula in ano. Radiology 2006; 239 : 18-33.

25. Kuijpers HC, Schulpen T. Fistulography for fistula in ano: is it useful? Dis Colon Rectum 1985; 28: 103-104.

26. Halligan S. Imaging fistula in ano. Clin Radiol 1998; 53: 85-95.

27. Sahni VA, Ahmad R, Burling D. Which method is best for imaging of perianal fistula? Abdom Imaging 2008; 33: 26-30.

28. Alabiso ME, Iasiello F, Pellino G, et al. 3D-EAUS and MRI in the activity of anal fistulas in Crohn's disease. Gastroenterol Res Pract 2016; 2016: 1895694.

29. Gecse KB, Bemelman W, Kamm MA, et al. World Gastroenterology Organization, International Organisation for Inflammatory Bowel Diseases IOIBD, European Society of Coloproctology and Robarts 
Clinical Trials; World Gastroenterology Organization International Organisation for Inflammatory Bowel Diseases IOIBD European Society of Coloproctology and Robarts Clinical Trials. A global consensus on the classification, diagnosis and multidisciplinary treatment of perianal fistulising Crohn's disease. Gut 2014; 63: 1381-1392.

30. Sahni VA, Ahmad R, Burling D. Which method is best for imaging of perianal fistula? Abdom Imaging 2008; 33: 26-30.

31. Gage KL, Deshmukh S, Majura KJ, et al. MRI of perianal fistulas: bridging the radiological-surgical divide. Abdom Imaging 2013; 38: 1033-1042.

32. Sheedy SP, Bruining DH, Dozois EJ, et al. MR Imaging of perianal Crohn disease. Radiology 2017; 282: 628-645.
33. Lunniss PJ, Barker PG, Sultan AH, et al. Magnetic resonance imaging of fistula-inano. Dis Colon Rectum 1994; 37: 708-718.

34. Maccioni F, Colaiacomo MC, Stasolla A, et al. Value of MRI performed with phased-array coil in the diagnosis and pre-operative classification of perianal and anal fistulas. Radiol Med (Torino) 2002; 104: 58-67.

35. Lunniss PJ, Armstrong P, Barker PG, et al. Magnetic resonance imaging of anal fistulae. Lancet 1992; 340: 394-396.

36. Halligan S, Bartram CI. MR imaging of fistula in ano: are endoanal coils the gold standard? AJR Am J Roentgenol 1998; 171: 407-412. 